\title{
Single RNA polymerases on the stage
}

Two different single-molecule assays allowed researchers to answer long-standing questions about Escherichia coli RNA polymerase (RNAP) elongation - its mechanism and $\sigma^{70}$ content.

To study a molecular motor, it is useful to observe how it moves along its track. Although this approach has been applied successfully to proteins like myosin and kinesin, the much smaller steps of RNAP - the enzyme responsible for transcription of all genes in E.coli—were beyond the resolution limit of those studies.

Now, Steven Block at Stanford University and his colleagues have improved an optical trapping system to attain the Angstromlevel resolution necessary to discern the base pair-sized steps of RNAP (Abbondanzieri et al., 2005). Traditional optical traps operate by computer feedback and thus are unstable and can introduce noise. By implementing a passive optical trap — in which one bead is placed in the nonlinear region at the edge of the trap-Block's group eliminated the need for computer feedback. "You can just dial up the light intensity to get whatever force you want," explains Block.

Block's group attached RNAP to one bead and the upstream end of a DNA template to another. The bead with the RNAP was held in the nonlinear region of an optical trap. As the enzyme moved along the DNA, this bead moved away from the DNA-tethered bead in the linear region of another optical trap, which was under constant force and thus did not move (Fig. 1). By monitoring this displacement under a microscope, the researchers observed RNAP in action and found that the enzyme takes $\sim 3.7-\AA$ steps, approximately the distance of one DNA base pair. Using this system, the authors attempted to resolve the mechanism by which RNAP moves. Their data support a 'ratchet' model in which the enzyme moves by brownian motion, and binding of the incoming NTP fixes it in the forward-translocated state.

This powerful method could be applied to other enzymes as well, and with increasing

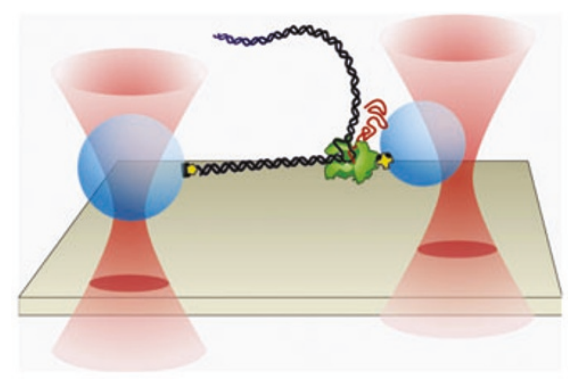

Figure $1 \mid$ The 'dumbbell' assay. A DNA molecule and RNAP are attached to polystyrene beads (blue). Optical traps (pink) hold the beads above the glass surface. (Image courtesy of S. Block.)

demand, Block believes that the technology they have built should become commercially available in the near future "because singlemolecule biophysics is growing by leaps and bounds right now. Originally people just applied it to motor proteins like myosin and kinesin, and now they are applying it to processive nucleic acid enzymes. And with Ångstrom-level resolution, you could apply it to any enzyme active site."

Single-molecule analysis is definitely popular: in another recent article, researchers asked whether the transcription initiation factor $\sigma^{70}$ is obligatorily released from RNAP at the transition from transcription initiation to elongation (Kapanidis et al., 2005). Shimon Weiss and colleagues at the University of California, Los Angeles, in collaboration with Richard Ebright's group at Rutgers University, labeled $\sigma^{70}$ with a donor fluorophore and the DNA template with an acceptor fluorophore, and developed a fluorescence resonance energy transfer (FRET)based single-molecule assay to monitor the labeled transcription complexes.

As individual complexes passed by diffusion through a femtoliter-scale confocal volume, they were illuminated by laser light that alternated between wavelengths that excite donor and acceptor fluorophores. From measurements of fluorescence, the researchers were able to calculate both fluorophore stoichiometry, which provided information about whether $\sigma^{70}$ was present in the complex, and FRET efficiency, which provided information about the location of the transcription complex on the DNA.

By performing this assay on both early and mature elongation complexes, they provide evidence that $\sigma^{70}$ release is not obligatory for transcription elongation. They determined that at the lacUV5 promoter, $~ 80 \%$ of the complexes contain $\sigma^{70}$ after transition to elongation, and just over half of the mature elongation complexes retain $\sigma^{70}$. These in vitro data suggest that $\sigma^{70}$ release is stochastic, rather than obligatory, and the authors propose that the release may be even more efficient in vivo.

"The main idea here is that we can measure distance and stoichiometry at the same time," says Weiss. An advantage of this assay over their previous approach is that it allows independent measurement of these variables and thus simplifies the analysis of heterogeneous populations of molecules. First author Achillefs Kapanidis adds that this method can even be extended to measure changes in three distances and three pairwise stoichiometries by adding a third-color probe.

Although this system was designed to assay the binding of a regulatory factor, Ebright points out that it can be used to address much more detailed questions about "how the polymerase active center translocates in initiation, pausing, editing and termination, when and how polymerase first breaks its interactions with promoter DNA and with activators, [and to define] changes in structure of polymerase, such as opening and closing of the active-center cleft." He adds: "These applications are the future of the technique." Irene Kaganman

\section{RESEARCH PAPERS}

Abbondanzieri, E.A. et al. Direct observation of base-pair stepping by RNA polymerase. Nature 438, 460-465 (2005).

Kapanidis, A.N. et al. Retention of transcription initiation factor $\sigma^{70}$ in transcription elongation: single-molecule analysis. Mol. Cell 20, 347-356 (2005). 